\title{
Apatinib-induced protective autophagy and apoptosis through the AKT-mTOR pathway in anaplastic thyroid cancer
}

\author{
Haoran Feng ${ }^{1,2}$, Xi Cheng ${ }^{1,2}$, Jie Kuang ${ }^{1,2}$, Lingxie Chen ${ }^{1,2}$, Stanley Yuen ${ }^{3}$, Minmin Shi ${ }^{1,2}$, Juyong Liang ${ }^{1,2}$,
} Baiyong Shen ${ }^{1,2}$, Zhijian Jin ${ }^{1,2}$, Jiqi Yan ${ }^{1,2}$ and Weihua Qiu ${ }^{1,2}$

\begin{abstract}
Apatinib, an inhibitor of vascular endothelial growth factor receptor-2, has been shown to promote anti-cancer action across a wide range of malignancies, including gastric, lung, and breast cancers. Our previous study showed that apatinib increases apoptosis in anaplastic thyroid carcinoma (ATC), but the direct functional mechanism of tumor lethality mediated by apatinib is still unknown. In this study, we demonstrated that apatinib induced both autophagy and apoptosis in human ATC cells through downregulation of p-AKT and p-mTOR signals via the AKT/mTOR pathway. Moreover, inhibition of apatinib-induced autophagy increased apatinib-induced apoptosis in ATC cells, and additional tumor suppression was critically produced by the combination of apatinib and the autophagy inhibitor chloroquine in vivo and in vitro. These findings showed that both autophagy and AKT/mTOR signals were engaged in ATC cell death evoked by apatinib. ATC patients might benefit from the new anti-cancer drug, and molecular targeted treatment in combination with autophagy inhibitors shows promise as a treatment improvement.
\end{abstract}

\section{Introduction}

Thyroid cancer is one of the most common malignant endocrine tumors. More than 50,000 new thyroid cancer cases would be diagnosed in 2018 , and the quantity of cases shows an obvious increasing trend ${ }^{1}$. The incidence of anaplastic thyroid carcinoma (ATC) is $1.5 \%$ in all thyroid cancers, and it is the major cause of all thyroid carcinoma-related deaths with a median survival time of 3-9 months due to high levels of extrathyroidal invasion, distant metastasis and resistance to conventional treatment $^{2-4}$. In all, ATC patients require more effective

\footnotetext{
Correspondence: Zhijian Jin (drjinzj@163.com) or Jiqi Yan (yanjiqi@aliyun.com) or Weihua Qiu (qwh11072@rjh.com.cn)

'Department of General Surgery, Ruijin Hospital, Shanghai Jiao Tong University School of Medicine, 200025 Shanghai, China

${ }^{2}$ Shanghai Institute of Digestive Surgery, Ruijin Hospital, Shanghai Jiao Tong University School of Medicine, 200025 Shanghai, China

Full list of author information is available at the end of the article.

These authors contributed equally: Haoran Feng, Xi Cheng, Jie Kuang

Edited by J. Martinez
}

therapy in addition to surgery and radioactive iodine therapy.

Apatinib, a small-molecule inhibitor of vascular endothelial growth factor receptor-2 (VEGFR-2), can induce apoptosis and suppress tumor proliferation in a variety of tumors $^{5-7}$. This drug has shown promising results in gastric cancer patients who failed standard chemotherapy $^{8,9}$. In addition, clinical trials that include breast cancer, esophageal cancer, colorectal cancer, liver cancer, and non-small cell lung cancer (NSCLC) are currently under investigation $^{10-14}$.

Apoptosis and autophagy are the two main mechanisms that cause programmed cell death $(\mathrm{PCD})^{15}$. Unlike apoptosis, autophagy is considered a double-edged sword that depends on the nature and cellular context of the stimuli ${ }^{16,17}$. The role of autophagy in cancer is complex. Under certain stress conditions, upregulation of autophagy might lead to cell death ${ }^{18,19}$. With the selective degradation of cellular components, autophagy also supplies a cell-

\section{(c) The Author(s) 2018}

(c) (i) Open Access This article is licensed under a Creative Commons Attribution 4.0 International License, which permits use, sharing, adaptation, distribution and reproduction cc) in any medium or format, as long as you give appropriate credit to the original author(s) and the source, provide a link to the Creative Commons license, and indicate if changes were made. The images or other third party material in this article are included in the article's Creative Commons license, unless indicated otherwise in a credit line to the material. If material is not included in the article's Creative Commons license and your intended use is not permitted by statutory regulation or exceeds the permitted use, you will need to obtain permission directly from the copyright holder. To view a copy of this license, visit http://creativecommons.org/licenses/by/4.0/. 
survival pathway, maintaining the recycling of nutrients and the generation of energy in all eukaryotes ${ }^{20-22}$.

In our previous study, we proved that apatinib could be a potential therapeutic strategy for ATC in vivo and in vitro ${ }^{23}$. However, the detailed regulation mechanism still needs further illustration. In this study, we confirmed that apatinib could induce autophagy and apoptosis through the AKT/mTOR pathway in ATC cells and that autophagy inhibition by chloroquine (CQ) could significantly enhance the anti-cancer effects of apatinib. These findings offer sequential and valid complementary evidence to our initial apatinib research.

\section{Materials and methods}

\section{Cell culture and compounds}

Human ATC cell lines KHM-5M and C643 were purchased from the China Center for Type Culture Collection (CCATCC, China). The C643 and KHM-5M cells were cultured in RPMI-1640 medium supplemented with $10 \%$ foetal bovine serum (Gibco, USA) at $37^{\circ} \mathrm{C}$ in $5 \% \mathrm{CO}_{2}$ (Shanghai Medical Instruments, China). Apatinib was obtained from Hengrui Medicine Co. Ltd. (Jiangsu, China), dissolved in DMSO and diluted with 1640 medium to the desired concentration with a final DMSO concentration of $0.1 \%$ for in vitro studies. Prior to each treatment, cells were plated overnight and displayed a similar subconfluently density at the time of drug exposure. The SC79, CQ, and rapamycin were purchased from Sigma-Aldrich Chemical Company (St. Louis, MO, USA) and were dissolved in PBS and diluted with RPMI-1640 to the desired concentration. Bafilomycin A1 (Baf A1) was obtained from Selleck Chemicals (Houston, TX, USA).

\section{Establishment of stable cell lines for autophagy analyses}

Lentiviral packaging was performed as previously described $^{24}$. In brief, $24 \mathrm{~h}$ prior to transfection, C643 and KHM-5M cells in the logarithmic growth phase were trypsinized and adjusted to $1.0 \times 10^{6}$ per $\mathrm{ml}$. The cells were reseeded into $15-\mathrm{cm}$ cell culture dishes and cultured for $24 \mathrm{~h}$ prior to transfection. The cells were $90-95 \%$ confluent on the day of transfection. Recombinant viral vectors encoding GFP-RFP- HLC3 (Jiman, China) were transfected into C643 and KHM-5M cells according to the manufacturer's instructions. After $48 \mathrm{~h}$, the expression of GFP and RFP was detected under an epifluorescence microscope (Olympics IX 71). Antibiotic-resistant colonies were selected on LB-puromycin agar plates for 2 weeks. After colony selection and further propagation, the stable cell line plasmid was maintained in RPMI-1640 (Sigma) at $37^{\circ} \mathrm{C}$.

\section{Cell viability assay and colony formation assay}

The cytotoxicity of apatinib was estimated using the CCK-8 assay (Cell Counting Kit-8, Dojindo, Japan).
Approximately 3000 cells were plated in 96-well plates and cultured in a $37^{\circ} \mathrm{C} / 5 \% \mathrm{CO}_{2}$ incubator for $24 \mathrm{~h}$ before treatment. The cells were treated with apatinib at $0,5,10$, 20,40 , and $80 \mu \mathrm{M}$ for 24,48 , or $72 \mathrm{~h}$, respectively. DMSO was added to the cultures as a solvent control. At the test point, $100 \mu \mathrm{l}$ Cell Counting Kit-8 (CCK-8) solution was added into each well, and the plate was incubated at $37^{\circ} \mathrm{C}$ for $2 \mathrm{~h}$ followed by OD detection using a spectrophotometer. For the colony formation assay, 1000 cells were plated in 6-well plates and cultured at $37^{\circ} \mathrm{C}$ for 2 weeks. The colonies were visualized by staining with $0.1 \%$ crystal violet in methanol for $30 \mathrm{~min}$. For the colony formation assay, 1000 cells suspended in $0.7 \%$ soft agar were plated on the surface of $1.2 \%$ soft agar in a 6-well plate and cultured at $37^{\circ} \mathrm{C}$ for 2 weeks.

\section{Confocal microscopy}

Cells were fixed with $4 \%$ paraformaldehyde (Sigma) for $20 \mathrm{~min}$. Subsequently, the cells were permeabilized with $0.1 \%$ Triton X-100 (Sigma) for $15 \mathrm{~min}$ at room temperature, washed with PBS and blocked with PBS containing $0.5 \%(\mathrm{w} / \mathrm{v})$ bovine serum albumin (BSA) and $0.15 \%(\mathrm{w} / \mathrm{v})$ glycine (BSA buffer) for $1 \mathrm{~h}$ at room temperature. The slices were treated with DAPI (Sigma) and visualized by confocal microscopy (Zeiss, LSM510).

\section{Cell transfection with siRNA}

ATG7 is essential for the autophagy conjugation system, autophagosome formation, and starvation-induced degradation of proteins and organelles in mammalian cells $^{25}$. Therefore, C643 and KHM-5M cells were pretreated with ATG7-siRNA interference to assess the contribution of autophagy. The ATG7-targeting sense sequence and the Universal Negative Control-siRNA were purchased from Invitrogen (12935-400, Invitrogen, Carlsbad, CA). The human ATG7 sequence (5'-GGAGA TCACAGCATCTATCCTT-3') was cloned into the $B a m H 1$ and $E c o$ R1 sites of the pGSU6-GFP vector (GTP600300, Genlantis, San Diego, CA). The siRNA plasmids were transfected using Lipofectamine 2000 (Invitrogen). Forty-eight hours after transfection, cells were collected via flow cytometry sorting. The irrelevant nucleotides did not target any annotated human genes and served as a negative control. The cells were cultured and treated by apatinib $(20 \mu \mathrm{M})$ for an additional $24 \mathrm{~h}$.

\section{Flow cytometry assay}

Flow cytometric assays of apoptosis were performed as previously described ${ }^{26}$. In brief, the Annexin V-FITC Apoptosis Detection kit (BD Pharmingen, USA) was used to detect the apoptosis of cells. Cells were treated with 0 , 10,20 , and $40 \mu \mathrm{M}$ apatinib for $24 \mathrm{~h}$. Both attached and the floating cells were harvested, washed twice with ice-cold PBS, and suspended in $100 \mu \mathrm{l}$ binding buffer. The cells 
were stained with $3 \mu \mathrm{l}$ Annexin V-FITC and $5 \mu \mathrm{l}$ propidium iodide (PI) and incubated at room temperature for 15 min in the dark. Finally, $300 \mu \mathrm{l} 1 \times$ binding buffer was added to each sample of cells. Apoptosis was analyzed by flow cytometry using the FACSCalibur system (BD Biosciences, USA).

\section{Electron microscopy}

After treatment, C643 and KHM-5M cells were immediately fixed in $2 \%$ glutaraldehyde and $2 \%$ paraformaldehyde in $0.1 \mathrm{~mol} / \mathrm{l}$ sodium phosphate buffer $(\mathrm{pH}$ 7.4) at $4{ }^{\circ} \mathrm{C}$ for $3 \mathrm{~h}$. The samples were embedded in a mixture of epon 618 and epoxypropane after post-fixing in $1 \%$ osmium tetroxide in the same buffer for $2 \mathrm{~h}$ and gradual dehydration in alcohols. Semi-thin sections were stained with toluidine blue, and ultrathin sections were stained with 5\% uranyl acetate and Reynold's lead citrate. Sections were examined on a Hitachi electron microscope equipped with a digital camera. For morphometric analysis, at least three independent experiments were performed.

\section{Western blotting}

After treatment, cells were harvested at various time intervals and digested in RIPA buffer in the presence of Protease Inhibitor (Pierce, USA) and Protein Phosphatase Inhibitor (New Cell \& Molecular Biotech, China) Cocktail. Total protein was extracted and quantified using BCA Protein Assay Kit (Pierce, Rockford, USA). Proteins were separated by SDS-PAGE and transferred to a PVDF membrane (Tanon, China). Blots were probed with antiPI3K (1:1000), anti-pPI3K (1:1000), anti-mTOR (1:1000), anti-p-mTOR (1:1000), anti-AKT (1:1000), anti-p-AKT (1:1000), anti-p62/SQSTM-1 (1:1000), anti-Beclin 1 (1:1000), anti-ATG7 (1:1000), anti-Bax (1:1000), anti-Bcl2 (1:1000), anti-PARP (1:1000), anti-Cleaved-caspase 3 (1:1000), anti-Caspase 3 (1:1000), anti-Cleaved-PARP (1:1000), anti-LC3 (1:1000) (all from Cell Signalling Technology, USA), and GAPDH (Abcam, 1:10,000) overnight at $4{ }^{\circ} \mathrm{C}$. GAPDH was used as the internal control. Goat anti-rabbit or goat anti-mouse horseradishperoxidase-conjugated IgG was used as the secondary antibody (Santa Cruz Biotechnology), and samples were incubated at room temperature for $1 \mathrm{~h}$. Bands were observed using enhanced chemiluminescence (Pierce). Band density was measured by densitometry, quantified using the public domain NIH Image J software (opensource Image J software is available at http://rsb.info.nih. gov/ij/) and normalized to an indicated sample in the identical membrane.

\section{In vivo $\mathrm{PC}$ xenograft tumor model}

Four-week-old male BALB/c nude mice were purchased from the Institute of Zoology, Chinese Academy of
Sciences of Shanghai. All experiments were performed in accordance with the official recommendations of the Chinese Zoological Society, and animals received humane care according to the criteria outlined in the "Guide for the Care and Use of Laboratory Animals". A suspension containing $1 \times 10^{6} \mathrm{KHM}-5 \mathrm{M}$ cells was subcutaneously injected into the right flanks of the nude mice. After $\sim 2$ weeks, when the tumors reached $\sim 5 \mathrm{~mm}$, all mice were randomly divided into four groups (4 in each group, group 1: Vehicle-only solution; group 2: CQ $60 \mathrm{mg} / \mathrm{kg}$; group 3: Apatinib $50 \mathrm{mg} / \mathrm{kg}$; and group 4: CQ $60 \mathrm{mg} / \mathrm{kg}+$ apatinib $50 \mathrm{mg} / \mathrm{kg}$ ). These compounds, dissolved in NS (normal saline solution), were administered via daily oral gavage per day. The tumor dimensions were measured using a Vernier caliper, and the tumor volume was calculated using the following formula: $V=\pi / 6 \times\left(W^{2} \times L\right)$. Tumor size and body mass were recorded twice per week. Three days after the last injection, the animals were killed by cervical decapitation, and tumors were removed and weighed. Samples were prepared for immunoblot analysis and TUNEL staining.

\section{Immunohistochemistry and TUNEL assay}

Immunohistochemistry analysis of xenograft tumors in nude mice was conducted as previous described ${ }^{27}$. In brief, tumor tissue sections with $4 \mu \mathrm{m}$ thickness were cut from paraffin-embedded tissue blocks, deparaffinized and rehydrated, and treated with $0.01 \mathrm{~mol} / \mathrm{l}$ citrate buffer (pH 6) for antigen retrieval. After inhibition of endogenous peroxidase activity for $30 \mathrm{~min}$ with methanol containing $0.3 \% \mathrm{H}_{2} \mathrm{O}_{2}$, the sections were incubated with rabbit anti-caspase-3 (1:200, Santa Cruz), and anti-Ki-67 (1:200, Santa Cruz) at $4{ }^{\circ} \mathrm{C}$ overnight. After incubation with HRP-labeled secondary antibody, apoptosis was identified using with the In Situ Cell Death Detection Kit, Fluorescein (Roche Applied Science, USA). Nuclei were detected by DAPI staining.

\section{Statistics}

Data are expressed as the means $\pm \mathrm{SD}$. Analysis of variance (ANOVA) and Student's $t$-test were chosen for comparison among groups. The Mann-Whitney $U$-test was applied in comparison of tumor volume. Categorical data were evaluated with the $\chi^{2}$ test or Fisher's exact test. $P$-values $<0.05$ were considered significant. Statistical analyses were processed using SPSS13.0 (SPSS Inc. Chicago, IL, USA).

\section{Results}

\section{Apatinib inhibits viability of ATC cells}

Our previous study showed that apatinib treatment could significantly inhibit the proliferation of ATC cell lines in vitro ${ }^{23}$. The IC-50 values for C643 and KHM-5M with apatinib were at the intermediate level of all ATC cell lines. Those data were established in our previous study, 


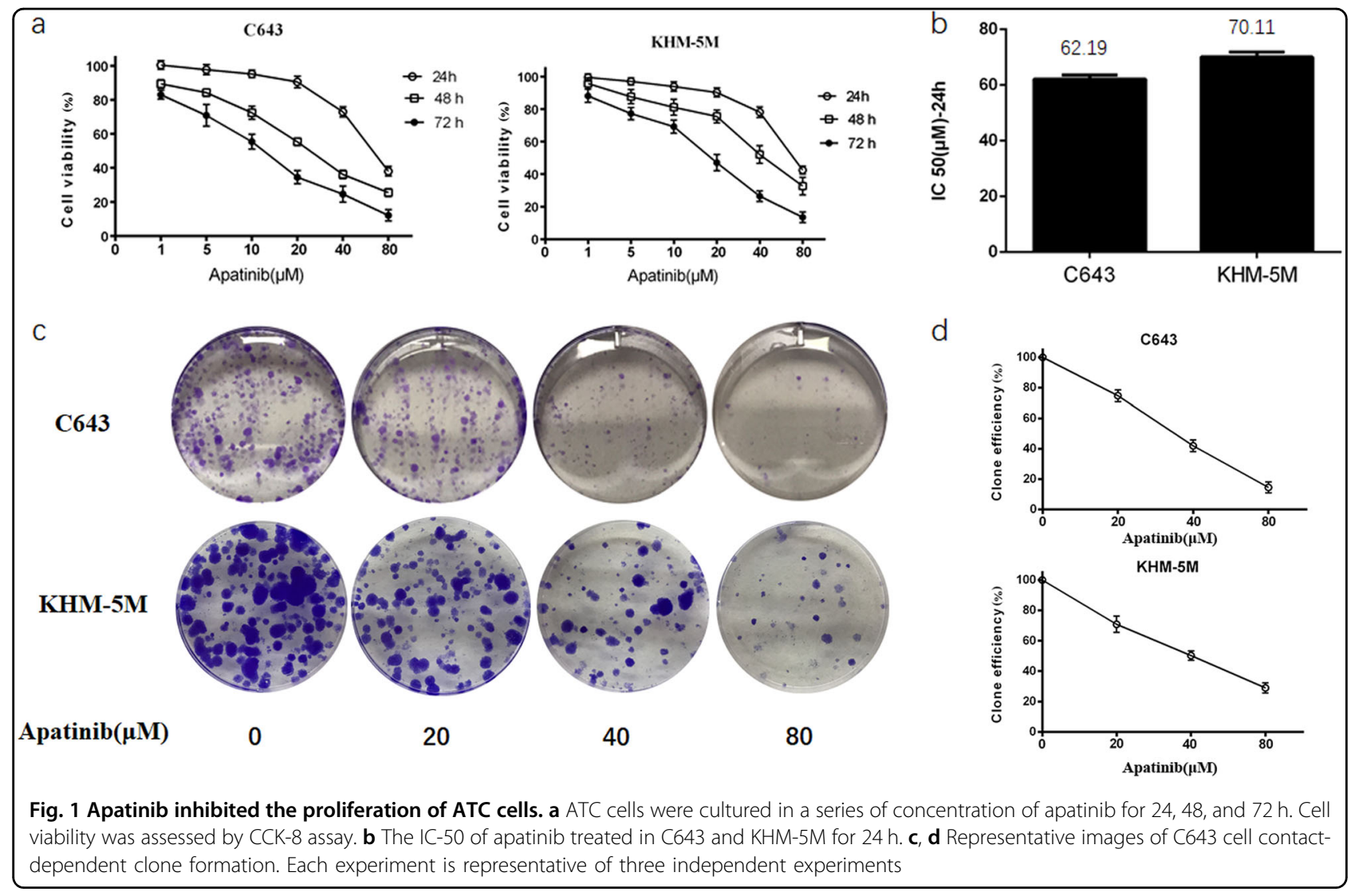

and the IC-50 values for C643 and KHM-5M were 62.68 and 70.83 for $24 \mathrm{~h}$, respectively ${ }^{23}$. In this study, C643 and KHM-5M were incubated with a series of concentrations of apatinib for 24,48 , and $72 \mathrm{~h}$. Apatinib concentrations ranging from 5 to $20 \mu \mathrm{M}$ did not significantly affect cell viability, whereas cytotoxicity increased dramatically at dosages greater than $40 \mu \mathrm{M}$ (Fig. 1a). Therefore, based on the CCK8 assay and to assess the role of apatinib-induced autophagy in ATC cells, the working concentration of apatinib was defined at $20 \mu \mathrm{M}$ for $24 \mathrm{~h}$ without resulting in obvious cell necrosis. The contact-dependent growth assay by cloning formation was performed to further confirm the effect of apatinib on inhibiting the proliferation of ATC cells. We identified fewer clone numbers in C643 and KHM-5M cells after apatinib treatment (Fig. 1c, d). Additionally, the decreasing trend in clone numbers is parallel to the concentration of apatinib treatment. When the apatinib concentration was greater than $40 \mu \mathrm{M}$, the cytotoxicity increased dramatically (Fig. 1b-d). All of these results confirmed the efficiency of apatinib in ATC cell inhibition and the IC-50 value in vitro.

\section{Increasing the apatinib level increases autophagosome formation}

Three different independent methods were used to examine autophagosome formation. First, western blot analysis was used to detect the expression of Beclin 1, LC3, ATG7, and P62. LC3 lipidation is a specific marker of autophagosomes in mammalian cells, and it is widely used in autophagy measurement. P62 is a hallmark of autophagic flux, and its expression level is closely associated with autophagy flux trigger and inhibition. Our results showed that the expression of LC3-II (LC3 lipidation), Beclin 1 and ATG7 were parallel to the increase in apatinib concentration, whereas P62 was consequently decreased (Fig. 2a, b). To further detect autophagic flux, Baf A1 was administered in ATC with or without apatinib. Our results showed p62 and LC3-II were increased with Baf A1/apatinib treatment than apatinib solo (Fig. 2e). Based on these results, we confirmed that apatinib could induce autophagosome formation in ATC cells in a dosedependent manner. In addition, GFP-RFP-hLC3 fluorescence was used to detect autophagic flux. The lentivirusencoding GFP-RFP-hLC3 fluorescent gene was transfected into ATC cells. The GFP-RFP-hLC3 fusion protein was dispersed in the cytoplasm, showing yellow-green spots (overlay of green and red fluorescence) under fluorescence microscopy. In autolysosomes, RFP was stably expressed with GFP quenching, and therefore, autolysosomes displayed red spots in contrast to yellowgreen spots. While autophagic flux is upregulated, yellow and red spots are both increased. Yellow spots are 


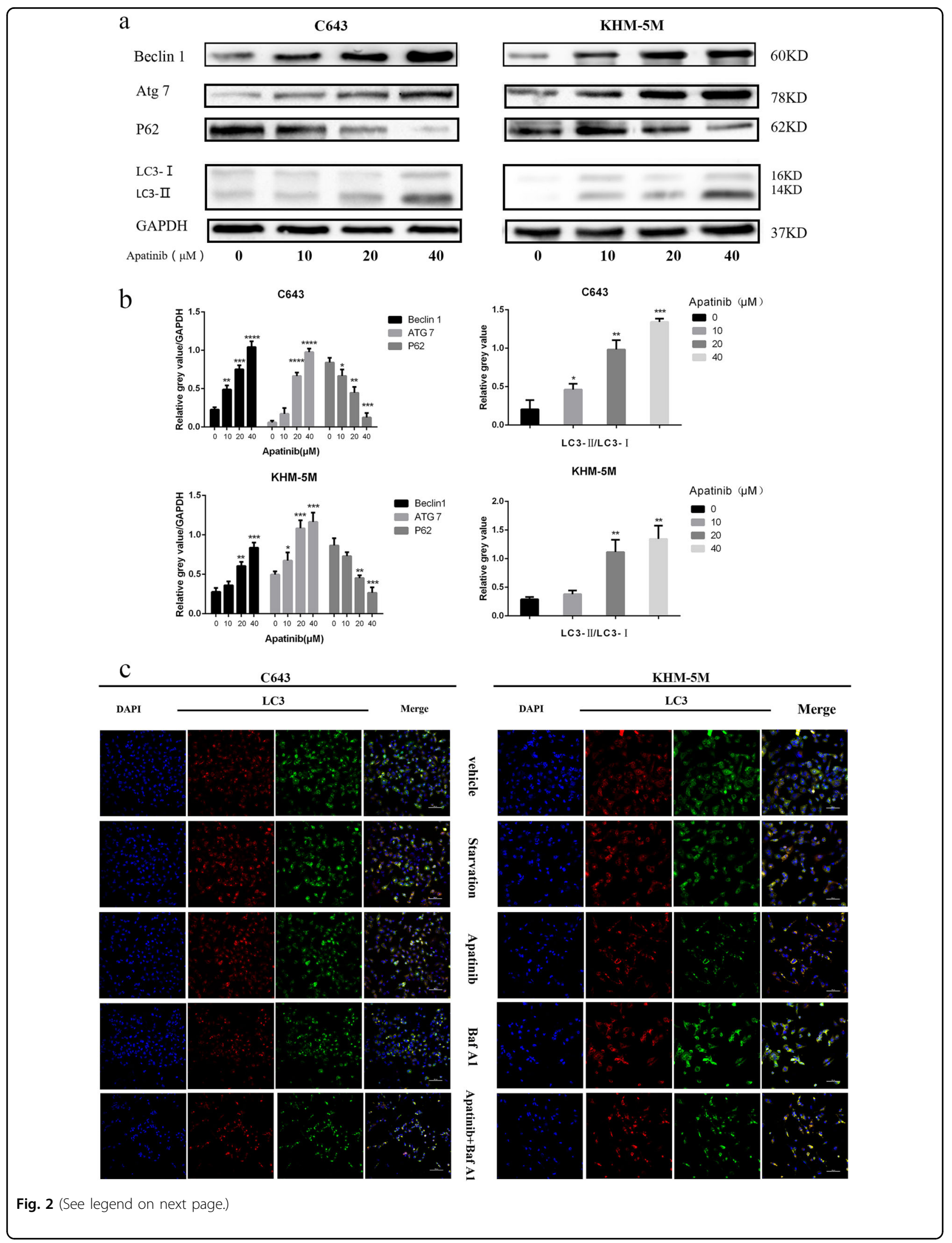




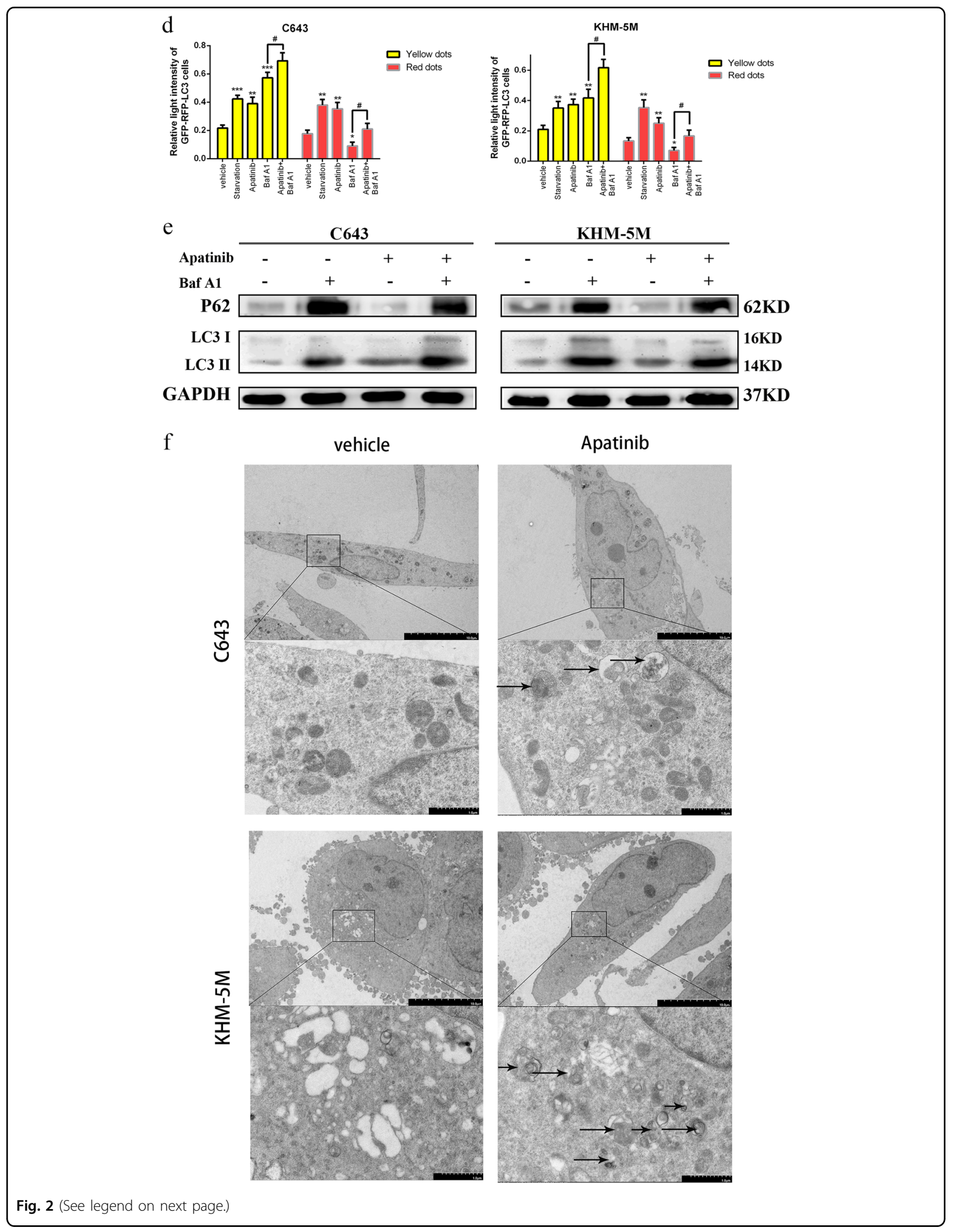


(see figure on previous page)

Fig. 2 Apatinib induced autophagy in ATC cells. a C643 and KHM-5M cells were treated with a serious concentration of apatinib for $24 \mathrm{~h}$. The Beclin 1, ATG7, P62, LC3-II, and GAPDH expressions were detected by western blot. b Densitometric analysis of the immunoblot reported in Fig. 2a. c Representative images of early-autophagosomes (yellow dots generated from overlapping GFP and RFP puncta) shown as yellow points and late autolysosomes (red dots generated from RFP puncta) shown as red points. C643 RFP-GFP-LC3 and KHM-5M RFP-GFP-LC3 cells (transfected with the RFP-GFP-hLC3 lentivirus) treated with $20 \mu \mathrm{M}$ apatinib for $24 \mathrm{~h}$, starvation or $50 \mathrm{nM}$ Baf A1 for $12 \mathrm{~h}$. $\mathbf{d}$ Densitometric analysis of the picture in Fig. $2 \mathrm{c}$. e ATC cells were treated as such Fig. 2c, the LC3, P62, and GAPDH were detected by western blot. f Electron microscopy showed C643 and KHM-5M cells in the presence of apatinib $(20 \mu \mathrm{M} / 24 \mathrm{~h}) .{ }^{*} p<0.05$; ${ }^{* *} p<0.01,{ }^{* *} p<0.001,{ }^{* * * *} p<0.0001$ compared with vehicle group. ${ }^{\#} p<0.05$; ${ }^{\# \#} p<0.01$, $\#$ \#\#\# $p<0.001, \# \#$ \# 20.0001 . Arrows: Autophaosomes/autolysosomes

increased only if the process of autophagosome fusion with lysosomes is inhibited. After treated with apatinib for $24 \mathrm{~h}$, confocal microscopy images showed that the numbers of both yellow dots and red dots increased more apparently in the apatinib group and starvation group than the vehicle group in C643 and KHM-5M cells. We observed that the yellow dots increased and red dots decreased, when the formation of autolysosome formation was inhibited by Baf A1. Yellow dots increased and red dots decreased in apatinib/Baf A1 group than apatinib alone treatment (Fig. 2c, d). Finally, electron microscopy images characteristically showed that ATC cells exposed to apatinib had a higher number of autophagosomes/ autolysosomes than the vehicle group (Fig. 2f). Thus, our data strongly suggested that apatinib induced autophagosome formation in ATC cell lines.

\section{Apatinib induces ATC cell apoptosis}

To evaluate the reason behind the growth inhibition induced by apatinib in ATC cells, western blot and flow cytometry were used to detect the level of apoptosis. As evidenced by the western blot analysis, the levels of cleaved-PARP, cleaved-caspase 3 and Bax expression were significantly increased with apatinib for $24 \mathrm{~h}$ in ATC cells, whereas $\mathrm{Bcl}-2$, PARP (total), and caspase 3 were consistently decreased (Fig. 3a, b). Flow cytometry was used to analyze the apoptotic cells after Annexin V-FITC and PI staining. Compared with the control group (apatinib $0 \mu \mathrm{M}$ ), treatment with apatinib significantly induced apoptosis in both C643 and KHM-5M cells. After $20 \mu \mathrm{M}$ apatinib treatment, values that were remarkably higher than those in the control group (C643: $12.23 \pm 0.51 \%$ vs. $5.26 \pm 0.89 \%, p<0.05$ and KHM-5M: $8.36 \pm 0.79 \%$ vs. 4.98 $\pm 1.42 \%, p<0.05$ ) (Fig. 3c, d). In addition, the apoptotic rate increased with respect to the concentrations of apatinib (C643: $24.91 \pm 1.1 \%$ (apatinib $40 \mu \mathrm{M}$ ), $12.23 \pm 0.51 \%$ (apatinib $20 \mu \mathrm{M}$ ), $8.08 \pm 0.67 \%$ (apatinib $10 \mu \mathrm{M}$ ), $5.26 \pm$ $0.89 \%$ (apatinib $0 \mu \mathrm{M}$ ); and KHM-5M: $12.3 \pm 1.34 \%$ (apatinib $40 \mu \mathrm{M}$ ), $8.36 \pm 0.79 \%$ (apatinib $20 \mu \mathrm{M}$ ), $5.62 \pm 0.59 \%$ (apatinib $10 \mu \mathrm{M}$ ), $4.98 \pm 1.42 \%$ (apatinib $0 \mu \mathrm{M}$ )). Taken together, our results demonstrated that apatinib was able to induce ATC cell apoptosis in a dose-dependent manner.

\section{The AKT/mTOR signal pathway affects apatinib-induced autophagy and apoptosis}

Many reports have suggested that the AKT-mTOR signaling pathway is a major negative regulator of apoptosis and autophagy ${ }^{28,29}$. Western blot results showed that pPI3K, p-AKT and p-mTOR decreased as a result of treatment by $20 \mu \mathrm{M}$ apatinib for $24 \mathrm{~h}$ in both C643 and KHM-5M cells, despite the unchanged total amount of PI3K, AKT and mTOR proteins (Fig. 4a, b). To further detect whether the AKT/mTOR pathway is involved in apatinib-induced autophagy and apoptosis in ATC cells, C643 and KHM-5M were subsequently treated together with apatinib and SC79 (an activator of AKT). As shown in Fig. 4c, co-treatment with apatinib and SC79 significantly reduced Bax, cleaved-PARP, and LC3 expression, whereas p-AKT, p-mTOR, Bcl-2, and P62 expression increased, indicating apoptosis and autophagy process inhibited when compared with apatinib treatment alone (Fig. 4c, d). Flow cytometry data showed that cells treated with apatinib and SC79 have a lower apoptotic rate than cells treated with apatinib alone (C643: $7.95 \pm 0.76 \%$ vs. $12.14 \pm 0.9 \%, p<0.05$; and KHM-5M: $6.21 \pm 0.68 \%$ vs. $9.18 \pm 0.78 \%, p<0.05$, Fig. 4e, f). Hence, activation of AKT via SC79 treatment could restore the autophagy and apoptosis induced by apatinib. Furthermore, rapamycin, an inhibitor of mTOR, was used to verify the involvement of mTOR in the autophagy and apoptosis induced by apatinib. The increasing expression of LC3 lipidation and cleaved-PARP induced by apatinib could be further enhanced by rapamycin treatment (Fig. 4g, h). Flow cytometry shows that ATC cells treated with apatinib and rapamycin had a higher apoptotic rate than apatinib alone (C643: $14.08 \pm 0.69 \%$ vs. $12.08 \pm 0.69 \%, p<0.05$; and KHM-5M: $\quad 10.54 \pm 0.55 \%$ vs. $8.66 \pm 0.37 \%, \quad p<0.05$ ) (Fig. 4i). AKT siRNA was used to knockdown AKT expression in C643 and KHM-5M cells, the expression of LC3 lipidation and cleaved-PARP in control group were lower than AKT siRNA group with or without Apatinib, respectively (Fig. 4j, k). Flow cytometry data showed that apoptotic rates in cells treated with apatinib in AKT siRNA group were greater than those in cells treated with apatinib in control-siRNA group (C643 control-siRNA/ apatinib group vs. AKT siRNA/apatinib group $12.94 \pm$ 


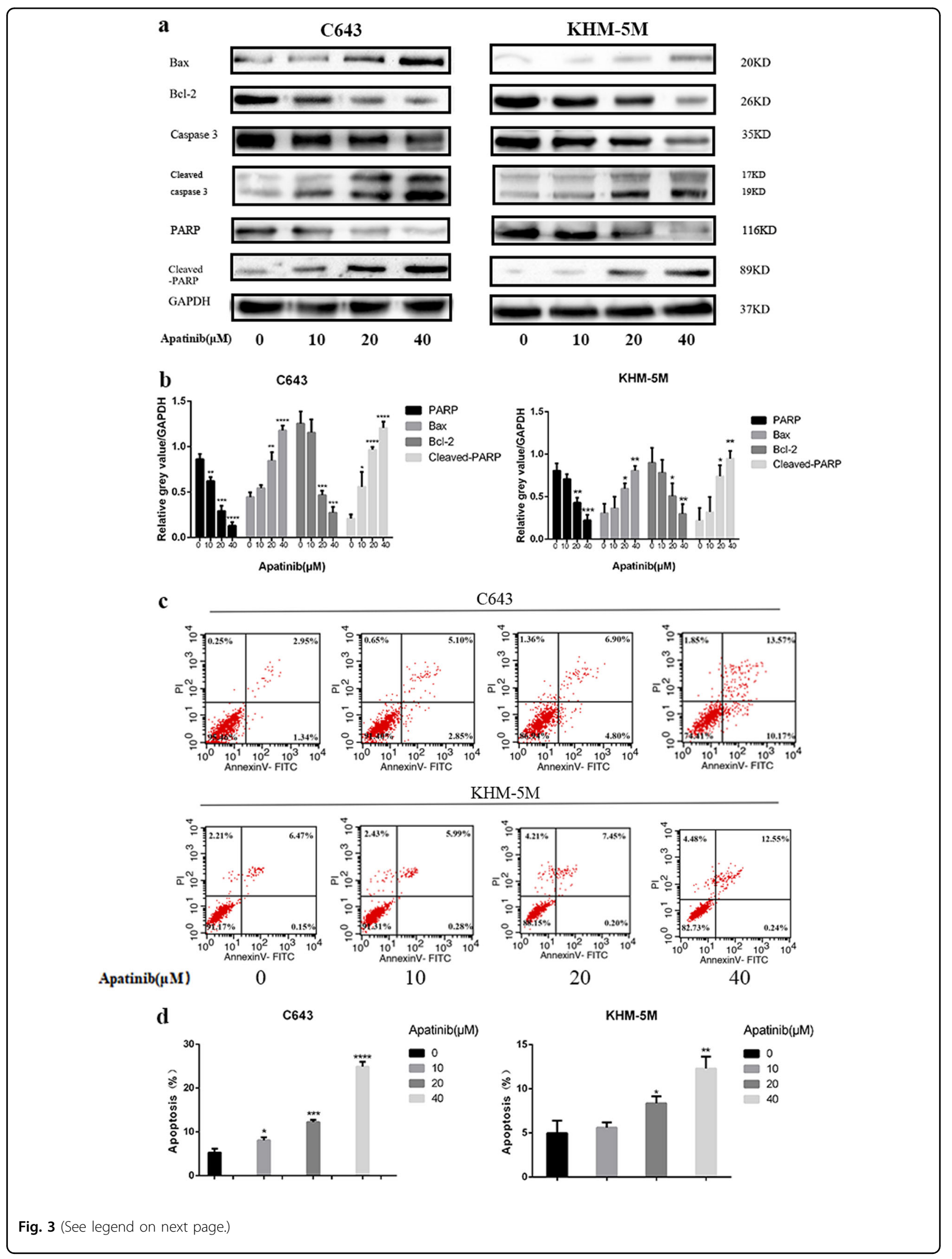


(see figure on previous page)

Fig. 3 Apatinib promotes apoptosis in ATC cells. a C643 and KHM-5M cells were treated with a serious concentration of apatinib for $24 \mathrm{~h}$. The caspase 3, cleaved-caspase 3, PARP, cleaved-PARP, Bcl-2, Bax, and GAPDH expressions were detected by western blot. b Quantification of relative gray value of bands compared with GAPDH, as detected by Fig. 3a. c C643 and KHM-5M cells were treated with the indicated concentrations for $24 \mathrm{~h}$. The ratio of apoptotic cells was measured by Annexin V-FITC and propidium iodide (PI) staining. The results were representative of three independent experiments. d As detected by Fig. 3c, (AnV+) (PI-) cells were considered early apoptotic and (AnV+) (PI+) cells were considered late apoptotic. The columns represent the mean \pm SD of the three independent experiments. ${ }^{*} p<0.05,{ }^{* *} p<0.01,{ }^{* *} p<0.001,{ }^{* * *} p<0.0001$ compared with control group

$0.57 \%$ vs. $16.33 \pm 1.13 \%, p<0.05$; and KHM-5M controlsiRNA/apatinib group vs. AKT siRNA/apatinib group $8.82 \pm 0.60 \%$ vs. $12.04 \pm 0.84 \%, p<0.05$ ) (Fig. 4l). Thus, our results confirmed that the AKT/mTOR signaling pathway could have a vital role in apatinib-induced apoptosis and autophagy regulation.

\section{Inhibition of autophagy enhances apatinib-induced apoptosis}

The paradoxical function of autophagy in the tumor might cause autophagic tumor cell death or adaptation to drug cytotoxicity, and hence, autophagy can support or inhibit the proliferation of tumor cells in different scenarios $^{18,19}$. CQ, an antimalarial lysosomal inhibitor, has been identified as an inhibitor of autophagy, which can prevent effective autophagic degradation and lead to accumulation of ineffective autophagosomes ${ }^{30,31}$. Both LC3 lipidation and P62 could be upregulated as a result of autophagosome accumulation due to the inhibition of the autophagy effective stage. As shown in Fig. 5a and b, the western blot results showed that CQ pre-treatment could increase LC3 lipidation and p62 as expected, indicating autophagy inhibition. Additionally, compared with apatinib solo treatment, apatinib combined with CQ pretreatment led to an increase of cleaved-PARP expression, revealing increased apoptosis in addition to autophagy inhibition. 3MA(3-Methyladenine) was also used to inhibit autophagy induced by apatinib in C643 and KHM$5 \mathrm{M}$ cell lines. Figure $6 \mathrm{c}$ and $\mathrm{d}$ showed the expression of p62 and cleaved-PARP were increased and LC3 lipidation was decreased with 3MA-apatinib treatment than apatinib solo. The results above illustrated that inhibition of autophagy induced by apatinib could enhanced apoptosis in ATC cells. Confocal microscopy images in Fig. 5e and $\mathrm{f}$ demonstrated fewer red spots and more yellow spots (autophagosome fusion with lysosomes is inhibited) in the apatinib with CQ pre-treatment group, and fewer yellow and red dots were observed in 3MA-apatinib group than apatinib group. Data from flow cytometry proved that apatinib plus pre-treatment with $C Q$ induced greater apoptosis rates (apatinib/CQ vs. apatinib: C643: $17.51 \pm$ $0.44 \%$ vs. $12.28 \pm 0.90 \%, p<0.05$; KHM-5M: $15.57 \pm 0.61 \%$ vs. $8.13 \pm 0.83 \%, p<0.05)$. Similarly, 3MA-apatinib treatment induced more apoptosis rate than apatinib single (apatinib/3MA vs. apatinib: C643: $15.98 \pm 0.51 \%$ vs. 12.28 $\pm 0.90 \%, p<0.05 ;$ KHM-5M: $13.92 \pm 0.50 \%$ vs. $8.13 \pm$ $0.83 \%, p<0.05$ ) (Fig. $5 g$, h). Altogether, our results indicated that apatinib, by means of CQ and 3MA treatment, could indeed enhance apoptosis together with autophagy inhibition. Furthermore, ATG7-siRNA was used to inhibit the early stage of autophagosome formation. The western blot results in Fig. 5i confirmed that the expression of ATG7 was diminished effectively in both KHM-5M and C643 cells. In addition to the p62 increase, the LC3 lipidation decrease demonstrated the special inhibition of autophagy induced by apatinib. Furthermore, following autophagy inhibition, cleaved-PARP expression was increased. Comparing apatinib with the control-siRNA group, flow cytometry proved that apatinib with ATG7siRNA induced greater apoptosis rates (apatinib/ATG7siRNA vs. apatinib: C643: $15.27 \pm 1.04 \%$ vs. $11.37 \pm 0.84 \%$, $p<0.05$; KHM-5M: $12.06 \pm 1.57 \%$ vs. $8.44 \pm 0.92 \%, p<$ $0.05)$, while without apatinib treatment, the apoptosis rates of both group had no obvious difference (ATG7siRNA vs. control-siRNA: C643: $4.06 \pm 0.79 \%$ vs. $4.05 \pm$ $0.65 \%, p>0.05$; KHM-5M: $4.53 \pm 0.51 \%$ vs. $4.32 \pm 0.46 \%$, $p>0.05$ ) (Fig. $5 \mathrm{i}-\mathrm{k}$ ). These results revealed that transfection with ATG7-siRNA could significantly enhance the apoptosis induced by apatinib in both C643 and KHM$5 \mathrm{M}$ cells. Thus, our results indicated that inhibiting the early stage of autophagosome formation not only could prohibit autophagy launch but could also enhance apoptosis in ATC cells. In all, although 3MA, CQ, and ATG7 could block autophagy activation in different steps, apoptosis could be promoted, which might be related to the deceleration of ATC cell proliferation.

\section{Tumor suppression induced by apatinib is enhanced by CQ}

Based on the results above, xenograft models were used to further confirm the effect of apatinib-mediated autophagy and apoptosis on tumor proliferation in vivo. After KHM-5M cells were subcutaneously injected into the right flanks, nude mice were divided into four groups: group $1=$ vehicle control, group $2=\mathrm{CQ}$ solo treatment, group $3=$ apatinib solo treatment, and group $4=$ apatinib with CQ treatment. The xenograft tumors were allowed to develop for 14 days after injection, and the corresponding treatment was started according to therapeutic comparison. At 26 days after treatment, all mice were sacrificed. As expected, the tumor volumes in the CQ 


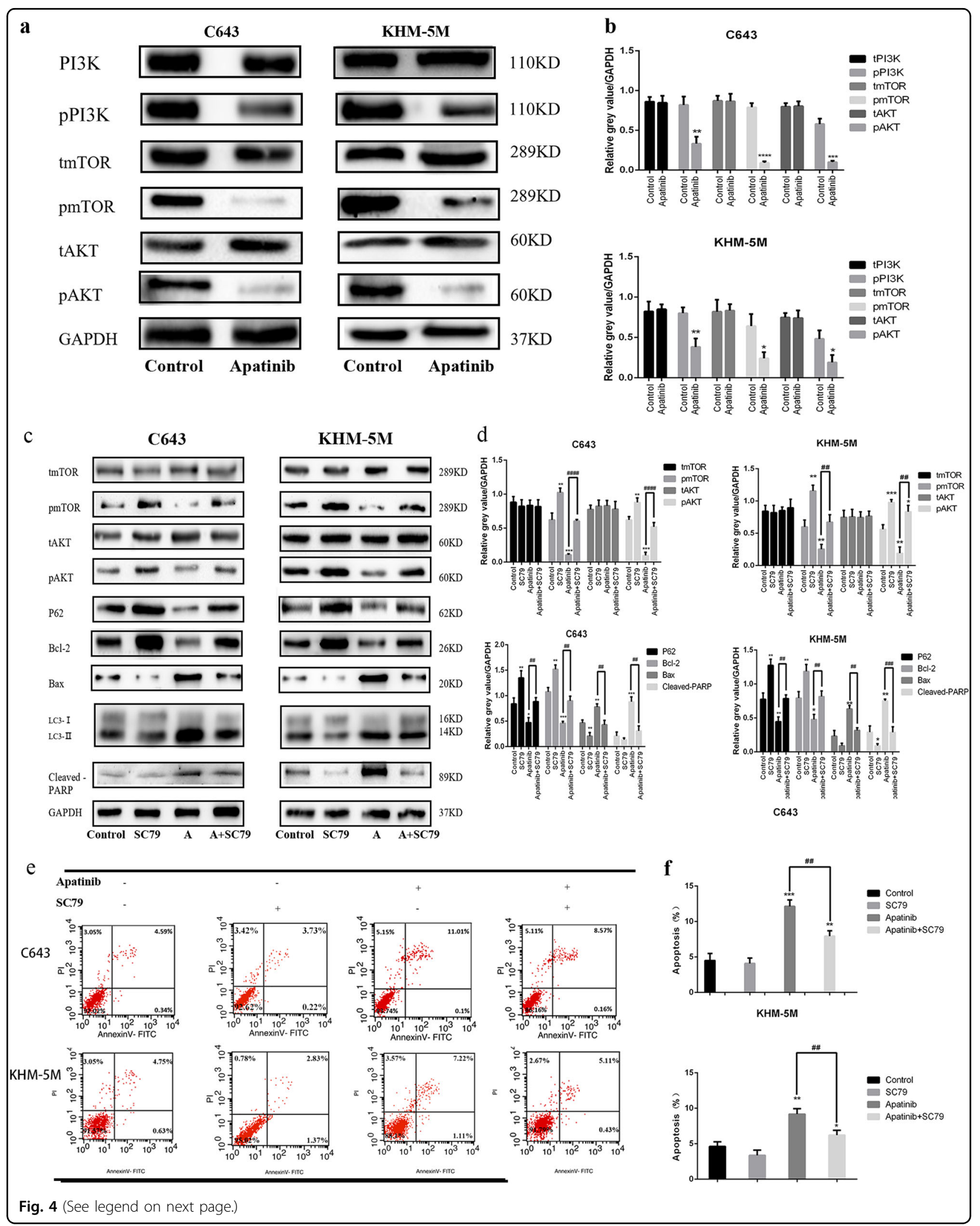




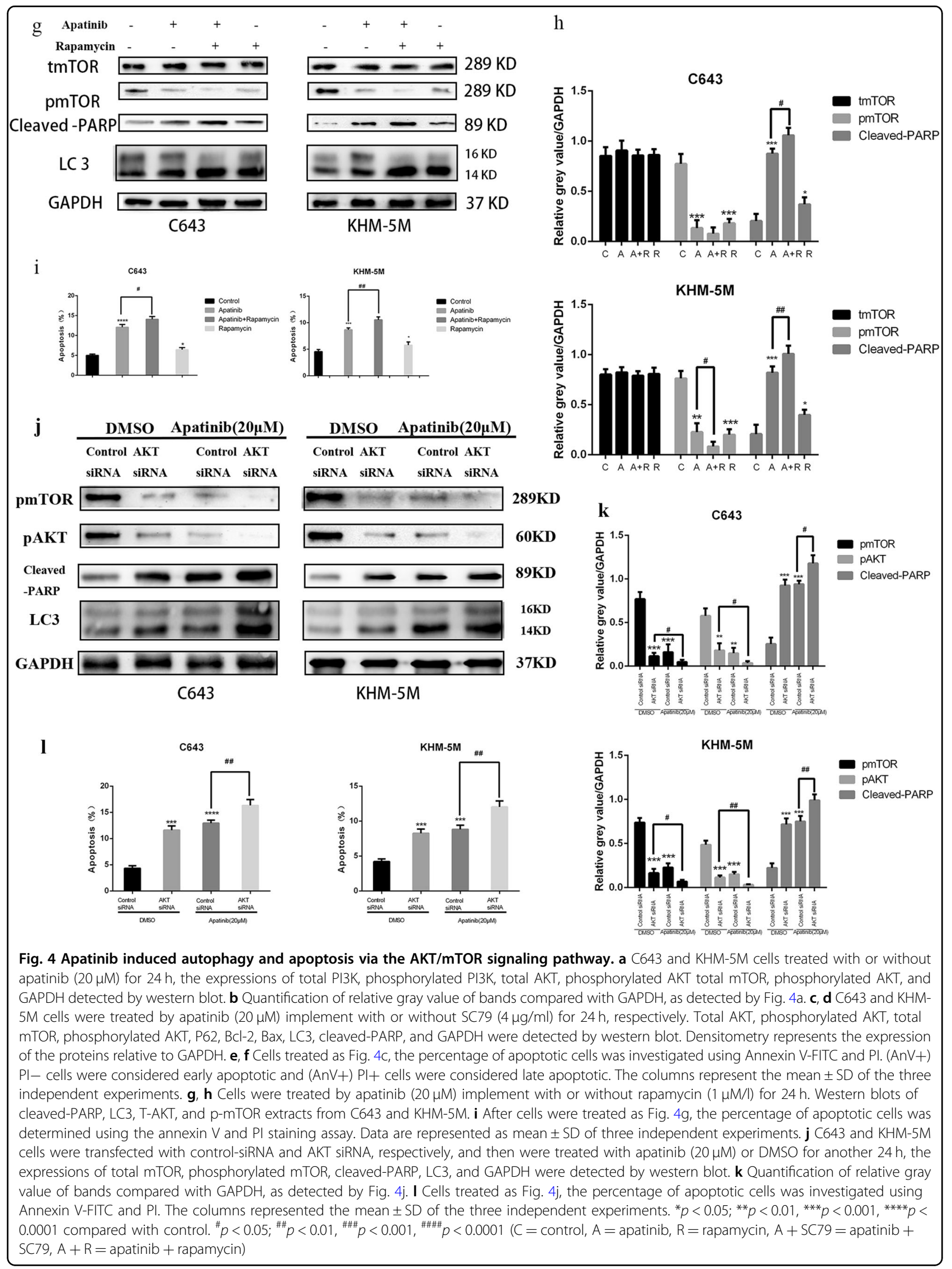




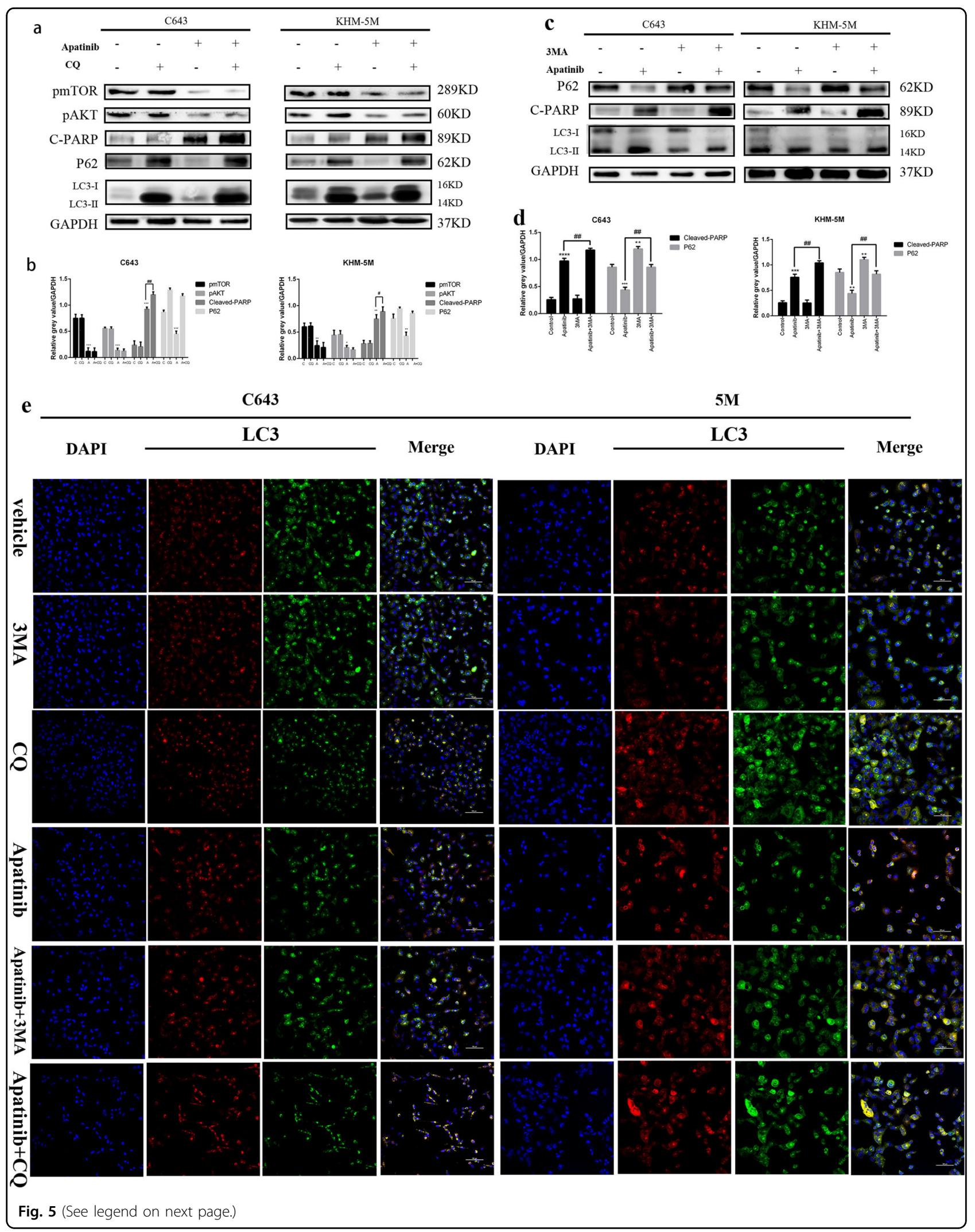




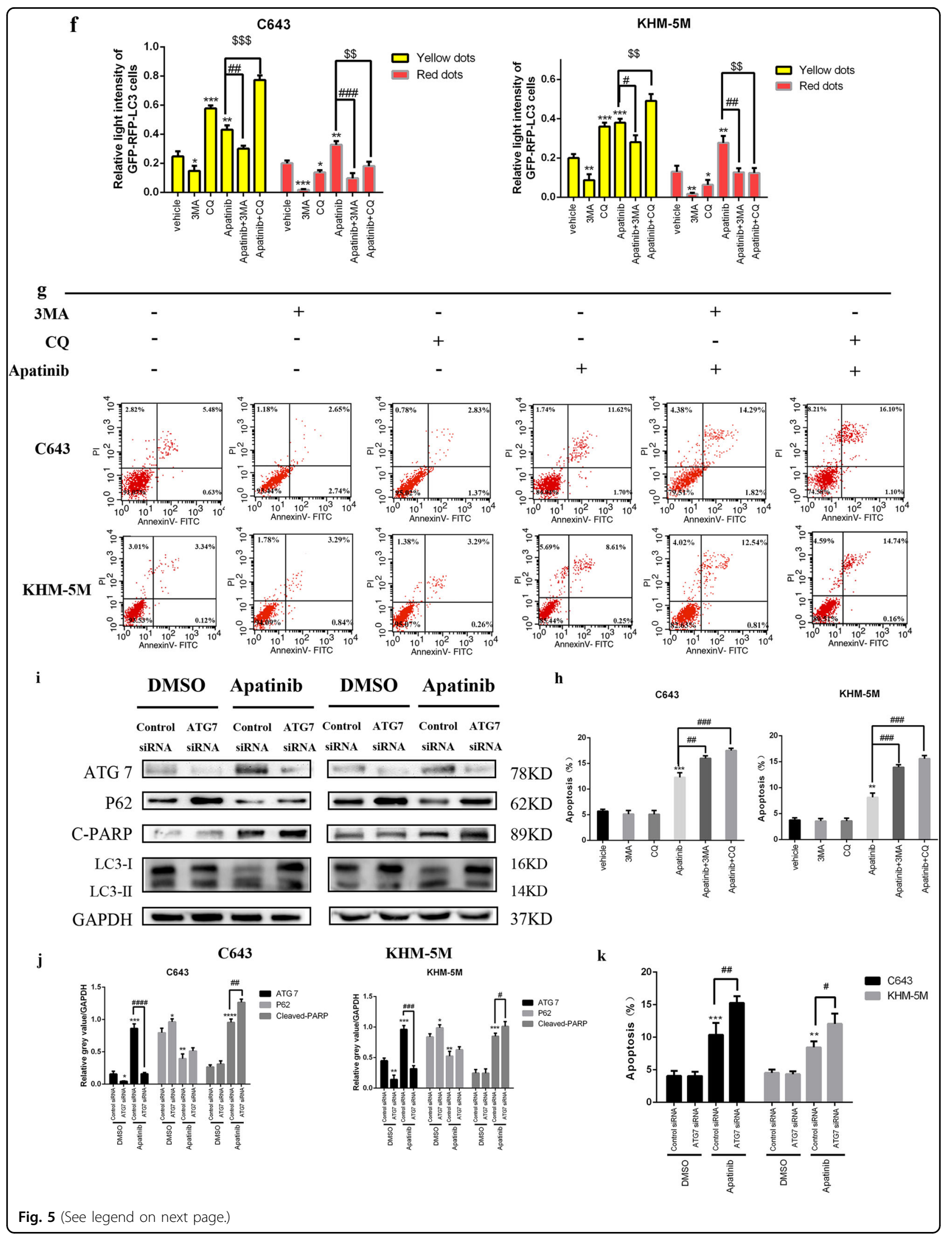


(see figure on previous page)

Fig. 5 Apatinib-induced autophagy protects ATC cells from apoptosis. a C643 and KHM-5M cells were pre-treated with CQ $(10 \mu \mathrm{M})$ for $6 \mathrm{~h}$, and then with apatinib $(20 \mu \mathrm{M})$ for another $24 \mathrm{~h}$, p-mTOR, p-AKT, cleaved-PARP, P62, LC3, and GAPDH were detected by western blot. b Quantification of relative gray value of bands compared with GAPDH, as detected by Fig. 5a. c C643 and KHM-5M cells were treated with apatinib for $24 \mathrm{~h}$ in the presence of $3 \mathrm{MA}(3 \mathrm{mM} / \mathrm{l})$, and then detected cleaved-PARP, LC3, P62, and GAPDH. $\mathbf{d}$ Quantification of relative gray value of bands compared with GAPDH, as detected by Fig. 5c. e As described in Fig. 2c, C643 RFP-GFP-LC3 and KHM-5M RFP-GFP-LC3 cells (transfected with the RFP-GFP-hLC3 lentivirus) were treated with or without apatinib for $24 \mathrm{~h}$ in either the presence or absence of $3 \mathrm{MA}$ and CQ. $\mathbf{f}$ Densitometric analysis of the picture in Fig. 5e. $\mathbf{g}$ Cells treated as Fig. 5e, the percentage of apoptotic cells was investigated using Annexin V-FITC and Pl. (AnV+) PI- cells were considered early apoptotic and (AnV+) PI+ cells were considered late apoptotic. The columns represent the mean \pm SD of the three independent experiments. $\mathbf{h}$ Cells treated as Fig. 5e, the percentage of apoptotic cells was determined using the annexin $\mathrm{V}$ and PI staining assay. Data are represented as mean \pm SD of three independent experiments. $\mathbf{i}$, $\mathbf{j}$ Western blot analyses showed the expression of ATG7, P62, cleaved-PARP, LC3, and GAPDH in C643/ATG7siRNA and KHM 5M/ATG7-siRNA treated with or without apatinib for $24 \mathrm{~h}$, compared with C643/control-siRNA and KHM 5M/ATG7-siRNA, respectively. i Data was shown as the percentage of apoptotic cells. Data were represented as mean \pm SD of three independent experiments. ${ }^{*} p<0.05 ;{ }^{* *} p<0.01$, ${ }^{* * *} p<0.001,{ }^{* * *} p<0.0001$ compared with control. ${ }^{\#} p<0.05 ;{ }^{\# \#} p<0.01,{ }^{\# \# \#} p<0.001$, ,\#\#\# $p<0.0001$ (c=control, A=apatinib, A+CQ=apatinib + CQ)

treatment did not show a significant difference (CQ vs. vehicle: $93 \pm 21.2 \mathrm{~mm}^{3}$ vs. $106.25 \pm 28.45 \mathrm{~mm}^{3}, p>0.05$ ) compared with those in the vehicle control group. The CQ combined with apatinib group exerted greater antitumor effects in KHM-5M xenograft tumor models compared with the drugs administered independently. (apatinib $+\mathrm{CQ}$ vs. apatinib: $12.25 \pm 7.68 \mathrm{~mm}^{3}$ vs. $29.75 \pm$ $11.32 \mathrm{~mm}^{3}, p<0.05$; apatinib + CQ vs. CQ: $12.25 \pm 7.68$ $\mathrm{mm}^{3}$ vs. $93 \pm 21.2 \mathrm{~mm}^{3} p<0.001$ ) (Fig. $6 \mathrm{a}-\mathrm{c}$ ). Consistent with the results of tumor volume, tumor weight could be suppressed by apatinib mono-therapy or CQ + apatinib combination therapy. Apatinib combined with CQ treatment showed a more effective inhibition (apatinib $+\mathrm{CQ}$ vs. apatinib: $13.48 \pm 8.44 \mathrm{mg}$ vs. $32.73 \pm 12.46 \mathrm{mg}, p<$ $0.05)$, and the percentage of growth inhibition in CQ group, apatinib group and $\mathrm{CQ}$ /apatinib group were $12.9 \%$, $72.1 \%$, and $88.5 \%$, respectively (Fig. 6b). Moreover, the expressions of Ki-67, and cleaved-caspase 3 in xenografts were detected by immunohistochemistry, and cell apoptosis was assayed by TUNEL. Compared with any other group, the expressions of Ki-67 was significantly reduced in the apatinib/CQ group, and the levels of cleavedcaspase 3 were significantly increased in the apatinib/CQ group. These results were further confirmed by the TUNEL assay (Fig. 6d, e). Indeed, the apatinib/CQ group had the greatest effect of tumor suppression among the four groups. All results showed that inhibited autophagy could enhance the effect of apatinib-induced growth inhibition in ATC cells in vivo.

\section{Discussion}

Although ATC has a rather low incidence, it was always characterized with a high risk of recurrence and poor prognosis. Traditional surgical treatment has been proven ineffective for ATC. Standard chemotherapy and targeted therapy were considered as the only palliative treatment method, but they did not prolong the 5-yr survival rate significantly ${ }^{2-4,32}$. Apatinib, a highly selective tyrosine kinase inhibitor of VEGFR-2, was proven to have an antitumor effect in various tumors ${ }^{5-7,33}$. Recently, apatinib has demonstrated a promising therapeutic effect for radioiodine refractory differentiated thyroid cancer ${ }^{34}$, which suggests a novel method for ATC treatment. We previously confirmed that apatinib could significantly reduce ATC angiogenesis in a dose- and time-dependent manner. Additionally, apatinib could decrease the ATC proliferation ability both in vivo and in vitro ${ }^{23}$. In addition to angiogenesis inhibition, in this study, apatinib could activate apoptosis and autophagy via suppression of the AKT/mTOR pathway, and apatinib-induced apoptosis could be enhanced by inhibiting apatinib-induced autophagy. This novel regulation mechanism could underline the anti-tumor effect of apatinib in ATC treatment.

Recent studies suggest that the autophagy and apoptosis signaling pathways can interact with each other ${ }^{34-38}$. Inhibition of autophagy could enhance apoptosis in osteosarcoma cells $^{39}$, and autophagy promotes apoptosis in mesenchymal stem cells under inflammatory microenvironment ${ }^{40}$. Therefore, understanding interaction of apoptosis and autophagy may be conducive to launch a new therapeutic strategy for ATC ${ }^{41}$. In our present study, we demonstrated that both autophagy and apoptosis in ATC cells could be enhanced by apatinib treatment. 3MA, CQ and ATG7-siRNA, chemical and genetic autophagy inhibitors, could, respectively, restrain autophagy in late phase and early phase; hence, they were employed to portrait the interactive relationship between autophagy and apoptosis. The results showed apatinib-induced autophagy could be effectively abolished by $3 \mathrm{MA}, \mathrm{CQ}$, and ATG7-siRNA. Consequently, blocking autophagy could promote apoptosis in ATC cell lines, which indicated that anti-proliferative effect of apatinib could be significantly boosted on autophagy inhibition. Thus, our study revealed the vital role of autophagy and apoptosis interaction in ATC therapeutic resistance.

The AKT signaling pathway is one of the major survival gateways of tumor cells, and growing evidence has showed that the over-active AKT/mTOR pathway promotes tumor cell survival ${ }^{42-45}$. As a downstream of 

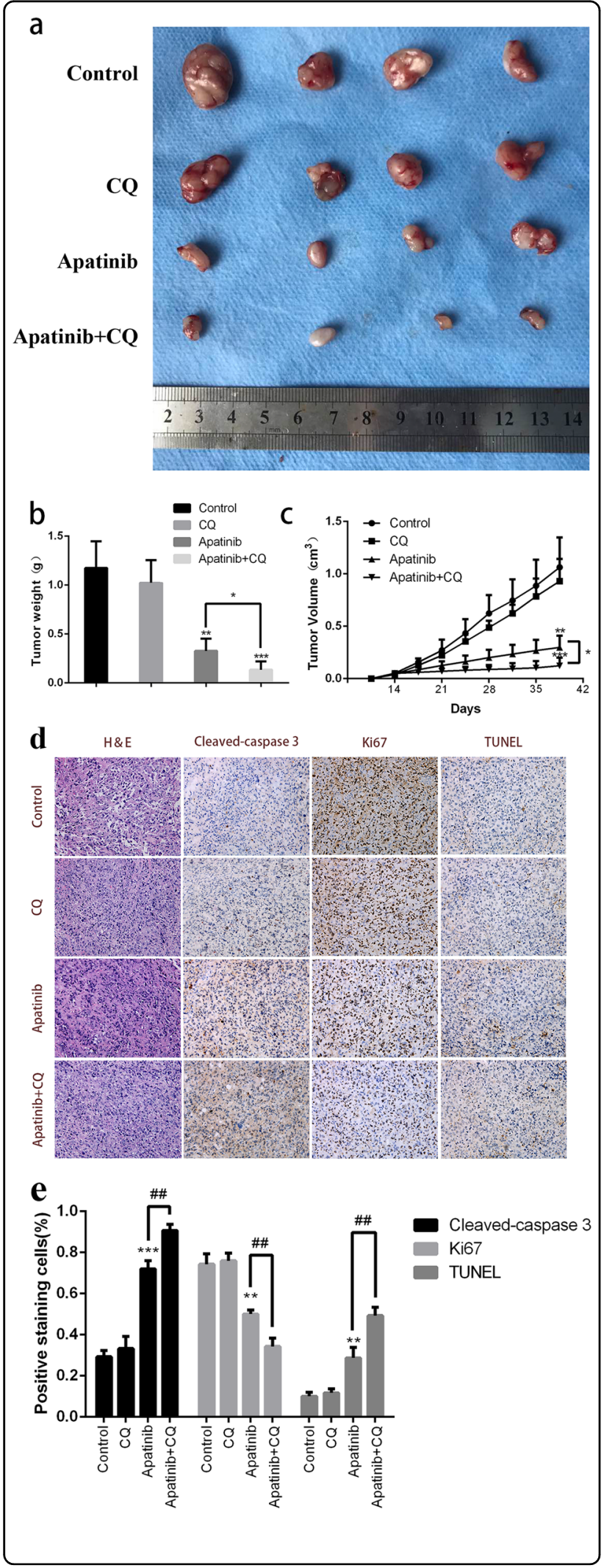

Fig. 6 Inhibition of tumor growth in vivo by apatinib and CQ. a Typical images of the xenograft tumors. $\mathbf{b}$ Average tumor weight for each group was calculated. c Xenograft tumor volumes were measured twice per week. $\mathbf{d}$ Representative images of IHC staining of Ki-67 and cleaved-caspase-3 were performed on serial sections of tumors from KHM-5M/Control, KHM-5M/CQ, KHM-5M/apatinib, and KHM-5M/apatinib plus CQ group, and cell apoptosis was assessed by TUNEL assay. e Densitometric analysis of the picture in Fig. $6 \mathrm{~d}$. ${ }^{*} p<0.05 ;{ }^{* *} p<0.01,{ }^{* * *} p<0.001,{ }^{* * *} p<0.0001$ compared with control. ${ }^{\#} p<0.05 ;{ }^{\# \#} p<0.01,{ }^{\# \# \#} p<0.001,{ }^{\# \# \# \#<0.0001}$

VEGFR, PI3K-AKT-mTOR signaling pathway is deeply involved in apoptotic effect of VEGFR-targeting therapy ${ }^{46,47}$. A promising therapeutic effect of apatinib in our current study has indicated the efficacy of VEGFR-2 inhibition in ATC (Supplementary material). Apatinib could decrease p-AKT and p-mTOR level in ATC cells. SC79, a special agonist for AKT, could restore the autophagy and apoptosis induced by apatinib in ATC. Similarly, rapamycin was used to reduce p-mTOR expression. Furthermore, AKT siRNA was used to knockdown the expression of AKT and p-AKT. Results above shown that apatinib could regulate the AKT/mTOR signaling pathway in ATC. Furthermore, inhibition of AKT-mTOR signaling pathway could directly stimulate autophagy and apoptosis ${ }^{48,49}$. In our study, apatinibinduced autophagy and apoptosis were decreased by SC79, and through downregulation the expression of pAKT by AKT siRNA, the autophagy and apoptosis induced by apatinib were enhanced. mTOR is mainly associated with apoptosis and autophagy. Rapamycin cotreatment has a modest increase in autophagy and apoptosis, but inhibiting autophagy with apatinib treatment will cause a greater increase. Thus, we confirmed that apatinib induce autophagy and apoptosis via AKT/ mTOR signaling pathway, and AKT/mTOR signaling pathway may closely associate with the growth inhibition effects of apatinib in ATC. Moreover, autophagy inhibitors as potential adjuvants to apatinib might represent an attractive therapeutic strategy for ATC treatment.

In conclusion, our data revealed a novel mechanism whereby apatinib induced apoptosis and autophagy via $\mathrm{AKT} / \mathrm{mTOR}$ pathway in ATC cell lines. Meanwhile blocking autophagy could enhance anti-tumor effect of apatinib in ATC cell lines both in vitro and in vivo. These findings validate that targeting autophagy with apatinib could be a promising therapeutic strategy for ATC.

\section{Acknowledgements}

We would like to acknowledge the Natural Science Foundation of China (NSFC: 81772558), Shanghai Charity Research Foundation of Cancer Research, Ph.D. Innovation Fund of Shanghai Jiaotong University School of Medicine 
(BXJ201709), and "Visiting Programs for Graduate Students of Shanghai Jiaotong University School of Medicine" for this study.

\section{Author details}

'Department of General Surgery, Ruijin Hospital, Shanghai Jiao Tong University School of Medicine, 200025 Shanghai, China. ${ }^{2}$ Shanghai Institute of Digestive Surgery, Ruijin Hospital, Shanghai Jiao Tong University School of Medicine, 200025 Shanghai, China. ${ }^{3}$ Biology Chemistry Major, University at Albany, New York, NY, USA

\section{Competing interest}

The authors declare that they have no conflict of interest.

\section{Publisher's note}

Springer Nature remains neutral with regard to jurisdictional claims in published maps and institutional affiliations.

Supplementary Information accompanies this paper at (https://doi.org/ 10.1038/s41419-018-1054-3).

Received: 23 April 2018 Revised: 28 August 2018 Accepted: 10 September 2018

\section{Published online: 09 October 2018}

\section{References}

1. Siegel, R. L., Miller, K. D. \& Jemal, A. Cancer statistics, 2018. CA Cancer J. Clin. 68 7-30 (2018)

2. Perri, F., Lorenzo, G. D., Scarpati, G. D. \& Buonerba, C. Anaplastic thyroid carcinoma: a comprehensive review of current and future therapeutic options. World J. Clin. Oncol. 2, 150-157 (2011).

3. Smallridge, R. C. \& Copland, J. A. Anaplastic thyroid carcinoma: pathogenesis and emerging therapies. Clin. Oncol. 22, 486-497 (2010).

4. Gervasi, R. et al. Thyroid surgery in geriatric patients: a literature review. BMC Surg. 12(Suppl 1), S16 (2012).

5. Lu, W. et al. Apatinib has anti-tumor effects and induces autophagy in colon cancer cells. Iran. J. Basic Med. Sci. 20, 990-995 (2017).

6. Zhang, H., Cao, Y., Chen, Y., Li, G. \& Yu, H. Apatinib promotes apoptosis of the SMMC-7721 hepatocellular carcinoma cell line via the PI3K/Akt pathway. Oncol. Lett. 15, 5739-5743 (2018).

7. Hu, X. et al. Multicenter phase II study of apatinib, a novel VEGFR inhibitor in heavily pretreated patients with metastatic triple-negative breast cancer. Int. J. Cancer 135, 1961-1969 (2014).

8. Zhang, S. Problematic analysis and inadequate toxicity data in phase III apatinib trial in gastric cancer. J. Clin. Oncol. 34, 3821 (2016)

9. Li, J. et al. Apatinib for chemotherapy-refractory advanced metastatic gastric cancer: results from a randomized, placebo-controlled, parallel-arm, phase I trial. J. Clin. Oncol. 31, 3219-3225 (2013).

10. Ding, L., Li, Q. J., You, K. Y., Jiang, Z. M. \& Yao, H. R. The use of apatinib in treating nonsmall-cell lung cancer: case report and review of literature. Medicine 95 e e3598 (2016).

11. Peng, $\mathrm{S}$. et al. Intracellular autocrine VEGF signaling promotes EBDC cell proliferation, which can be inhibited by apatinib. Cancer Lett. 373, 193-202 (2016).

12. Ter, Veer, E. et al. Second- and third-line systemic therapy in patients with advanced esophagogastric cancer: a systematic review of the literature. Cancer Metastas. Rev. 35, 439-456 (2016)

13. Zhou, N. et al. Response to apatinib in chemotherapy-failed advanced spindle cell breast carcinoma. Oncotarget 7, 72373-72379 (2016).

14. Langer, C. J., Mok, T. \& Postmus, P. E. Targeted agents in the third-/fourth-line treatment of patients with advanced (stage III/IV) non-small cell lung cancer (NSCLC). Cancer Treat. Rev. 39, 252-260 (2013).

15. Sperandio, S., de Belle, I. \& Bredesen, D. E. An alternative, nonapoptotic form of programmed cell death. Proc. Natl Acad. Sci. USA 97, 14376-14381 (2000).

16. Liu, J. J., Lin, M., Yu, J. Y., Liu, B. \& Bao, J. K. Targeting apoptotic and autophagic pathways for cancer therapeutics. Cancer Lett. 300, 105-114 (2011).

17. Su, M., Mei, Y. \& Sinha, S. Role of the crosstalk between autophagy and apoptosis in cancer. J. Oncol. 2013, 102735 (2013).
18. Codogno, P., Mehrpour, M. \& Proikas-Cezanne, T. Canonical and non-canonical autophagy: variations on a common theme of self-eating? Nat. Rev. Mol. Cell Biol. 13, 7-12 (2011).

19. Shintani, T. \& Klionsky, D. J. Autophagy in health and disease: a double-edged sword. Science 306, 990-995 (2004).

20. Levine, B. \& Klionsky, D. J. Development by self-digestion: molecular mechanisms and biological functions of autophagy. Dev. Cell 6, 463-477 (2004).

21. Marino, G., Niso-Santano, M., Baehrecke, E. H. \& Kroemer, G. Self-consumption: the interplay of autophagy and apoptosis. Nat. Rev. Mol. Cell Biol. 15, 81-94 (2014).

22. Hou, W., Han, J., Lu, C., Goldstein, L. A. \& Rabinowich, H. Autophagic degradation of active caspase-8: a crosstalk mechanism between autophagy and apoptosis. Autophagy 6, 891-900 (2010).

23. Jin, Z. et al. Apatinib inhibits angiogenesis via suppressing Akt/GSK3beta/ANG signaling pathway in anaplastic thyroid cancer. Cell Physiol. Biochem. 44 1471-1484 (2017).

24. $\mathrm{Du}, \mathrm{H}$. et al. Role of autophagy in resistance to oxaliplatin in hepatocellular carcinoma cells. Oncol. Rep. 27, 143-150 (2012).

25. Gonzalez, Y., Aryal, B., Chehab, L. \& Rao, V. A. Atg7- and Keap1-dependent autophagy protects breast cancer cell lines against mitoquinone-induced oxidative stress. Oncotarget 5, 1526-1537 (2014).

26. Cheng, $X$. et al. Pseudomonas aeruginosa-mannose-sensitive hemagglutinin inhibits pancreatic cancer cell proliferation and induces apoptosis via the EGFR pathway and caspase signaling. Oncotarget 7, 77916-77925 (2016).

27. Ma, D. et al. Leukemia inhibitory factor receptor negatively regulates the metastasis of pancreatic cancer cells in vitro and in vivo. Oncol. Rep. $\mathbf{3 6}$ 827-836 (2016)

28. Nicholson, K. M. \& Anderson, N. G. The protein kinase B/Akt signalling pathway in human malignancy. Cell Signal. 14, 381-395 (2012).

29. Majumder, P. K. \& Sellers, W. R. Akt-regulated pathways in prostate cancer Oncogene 24, 7465-7474 (2005).

30. Chen, P. M., Gombart, Z. J. \& Chen, J. W. Chloroquine treatment of ARPE-19 cells leads to lysosome dilation and intracellular lipid accumulation: possible implications of lysosomal dysfunction in macular degeneration. Cell Biosci. 1 10 (2011)

31. Hasanain, M. et al. alpha-Solanine induces ROS-mediated autophagy through activation of endoplasmic reticulum stress and inhibition of Akt/mTOR pathway. Cell Death Dis. 6, e1860 (2015).

32. Schlumberger, $M$. et al. Definition and management of radioactive iodinerefractory differentiated thyroid cancer. Lancet Diabet. Endocrinol. 2, 356-358 (2014).

33. Li, J. et al. Randomized, double-blind, placebo-controlled phase III trial of apatinib in patients with chemotherapy-refractory advanced or metastatic adenocarcinoma of the stomach or gastroesophageal junction. J. Clin. Oncol. 34, 1448-1454 (2016)

34. Lin, Y., Wang, C., Gao, W., Cui, R. \& Liang, J. Overwhelming rapid metabolic and structural response to apatinib in radioiodine refractory differentiated thyroid cancer. Oncotarget 8, 42252-42261 (2017).

35. Moretti, L., Cha, Y. I., Niermann, K. J. \& Lu, B. Switch between apoptosis and autophagy: radiation-induced endoplasmic reticulum stress? Cell Cycle 6 793-798 (2007).

36. Ryter, S. W., Mizumura, K. \& Choi, A. M. The impact of autophagy on cell death modalities. Int. J. Cell Biol. 2014, 502676 (2014)

37. Jing, K. \& Lim, K. Why is autophagy important in human diseases? Exp. Mol. Med. 44, 69-72 (2012).

38. Gump, J. M. \& Thorburn, A. Autophagy and apoptosis: what is the connection? Trends Cell Biol. 21, 387-392 (2011)

39. Liu, K. et al. Apatinib promotes autophagy and apoptosis through VEGFR-2 STAT3/BCL-2 signaling in osteosarcoma. Cell Death Dis. 8, e3015 (2017).

40. Dang, S. et al. Autophagy promotes apoptosis of mesenchymal stem cells under inflammatory microenvironment. Stem Cell Res. Ther. 6, 247 (2015).

41. Guo, J. Y., Xia, B. \& White, E. Autophagy-mediated tumor promotion. Cell 155 1216-1219 (2013)

42. Blume-Jensen, P. \& Hunter, T. Oncogenic kinase signalling. Nature 411 355-365 (2001).

43. Pene, F. et al. Role of the phosphatidylinositol 3-kinase/Akt and mTOR/P70S6kinase pathways in the proliferation and apoptosis in multiple myeloma Oncogene 21, 6587-6597 (2002). 
44. LoPiccolo, J., Blumenthal, G. M., Bernstein, W. B. \& Dennis, P. A. Targeting the PI3K/Akt/mTOR pathway: effective combinations and clinical considerations. Drug Resist. Update 11, 32-50 (2008).

45. $\mathrm{Hu}, \mathrm{H}$. Z. et al. Oridonin induces apoptosis via PI3K/Akt pathway in cervical carcinoma HeLa cell line. Acta Pharmacol. Sin. 28, 1819-1826 (2007).

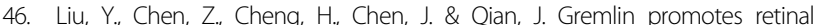
pigmentation epithelial (RPE) cell proliferation, migration and VEGF production via activating VEGFR-2-Akt-mTORC2 signaling. Oncotarget 8 979-987 (2017).
47. Peng, $H$. et al. Apatinib inhibits VEGF signaling and promotes apoptosis in intrahepatic cholangiocarcinoma. Oncotarget 7, 17220-17229 (2016).

48. Chen, Z. et al. LY294002 and rapamycin promote coxsackievirus-induced cytopathic effect and apoptosis via inhibition of PI3KAKT/mTOR signaling pathway. Mol. Cell. Biochem. 385, 169-177 (2014).

49. Luo, C. L. et al. The effect of quercetin nanoparticle on cervical cancer progression by inducing apoptosis, autophagy and anti-proliferation via JAK2 suppression. Biomed. Pharmacother. 82, 595-605 (2016). 